\title{
Wind Field Synthesis for Animating Wind-induced Vibration
}

\author{
Oyundolgor Khorloo ${ }^{1}$, Zorig Gunjee ${ }^{2}$, Batjargal Sosorbaram ${ }^{3}$ and Norishige Chiba ${ }^{3}$ \\ ${ }^{1}$ Graduate School of Engineering, Iwate University, Japan \\ 2 ECM Co., Ltd, Mongolia \\ ${ }^{3}$ Iwate University, Japan
}

\begin{abstract}
We present a simple method to generate three-dimensional frozen and non-frozen turbulent wind fields for use in the animation of wind-induced motion. Our approach uses $1 / \mathbf{f}^{\beta}$ noise to match the characteristics of natural wind. By employing a noise-based approach, the complexity as well as computational cost is reduced. Additionally, by considering key characteristics of actual wind that are applied in the structural engineering field, our proposed method is able to produce plausible results in outdoor wind field simulations. In this paper, we describe the implementation results of our proposed method and compare them with other existing approaches used to construct turbulent wind fields. The implementation and visualization are carried out for both two- and three-dimensional scenarios and compared with the results of other well-known methods.
\end{abstract}

Index Terms-1/f $\mathbf{f}^{\beta}$ noise, natural phenomena, stochastic modeling, wind field, wind turbulence.

\section{INTRODUCTION}

The mathematical modeling of wind is a challenging task because it involves a high degree of complexity. In the field of computer animation, modeling a wind field essentially depends on visual appearance and not on strict physical accuracy. Therefore, early methods considered developing approximations that could mimic the visual behavior of wind.

There have been several approaches to modeling the wind field in the computer graphics community. To depict animated trees and grass, Shinya and Fournier [1] constructed a complex flow field by modeling the wind field in Fourier space and then converting it to a time-varying force field. Similarly, Stam and Fiume [2] synthesized stochastic wind fields by applying a different spectrum filter to the turbulent motion of gas. Ota et al. [3] and $\mathrm{Hu}$ et al. [4] simulated the motion of branches and leaves swaying in a wind field by using noise functions. The most accurate wind field simulations are generated by using Navier-Stokes equations [5, 6]. Wejchert and Haumann [7] developed an aerodynamic model to simulate leaves falling from trees in a wind field. In addition, many other papers, such as [8-11], are concerned with modeling the faithful motions to the influence of external forces such as caused by a wind field.
In the animations of waving phenomena, Fujimoto et al. [12] used fractional Brownian motion (fBm). Qiang et al. [13] expanded 2D simulation of breaking waves into $3 \mathrm{D}$ representation by giving motion variation using $\mathrm{fBm}$.

Most approaches typically use stochastic approximations for wind simulation and carry out the simulation in the frequency domain. To realistically model natural phenomena, the filter should be based on real-world data. Although alternative approaches based on physical methods provide physical accuracy and visually convincing results, their computational cost is quite high. Methods based on noise functions are simpler than the abovementioned methods, but the simulated wind fields are not realistic enough because the key characteristics of actual wind are not considered. In contrast, in the approaches of [1] and [2], experimental data and statistics are borrowed from other fields such as structural engineering and oceanography and introduced to the graphics community to create synthetic images.

The aim of this study is the identification of an alternative approach for simulating a wind field and the animation of dynamic natural scenes with reduced computational costs while simultaneously producing a realistic visual simulation. We employ a noise-based approach to minimize computation time and stochastic approaches to approximate the complicated motion of specific phenomena. Fractional Brownian motion is one useful technique to represent natural objects or phenomena with randomness. Up to now, $\mathrm{fBm}$ has been mainly used for representing the shapes of static objects, such as mountainous terrains or clouds of fractal density distribution [14]. FBm is also known as $1 / \mathrm{f}^{\beta}$ noise. In our approach, we implement fBm functions using a simple spectrum synthesis technique based on FFT [15], but it is also possible to use other techniques such as Perlin noise [16] and procedural noise functions [17]. By using the main wind characteristics applied in structural engineering, we were able to control noise data more intuitively. Hence, our contributions can be summarized in the following points.

- Our method is simple but can produce wind fields of almost same quality as those of well-known approaches.

- $\quad$ Our proposed approach is comparable to other well-known approaches used in the computer graphics community.

- Wind fields simulated by our method can be described using relatively few parameters. 
we discuss the key characteristics of wind considered in the structural engineering field. In this section, some experimental data is included to illustrate the natural characteristics of wind predicted in an intuitive manner. Then, in Sections III and IV, we introduce our proposed two-dimensional and three-dimensional wind field model by comparing it to well-known methods. Implementation and visualization results are described in Section V. Finally, we summarize this paper in the Conclusion section.

\section{CHARACTERISTICS OF WIND}

Wind is a fairly common phenomenon and is considered the primary cause of motion in almost all outdoor simulations. Without a turbulent wind field, animations would lack a natural chaotic look, which is an important characteristic of most natural phenomena.

Here, we describe some fundamental characteristics and laws used as the theoretical basis of our research.

Closer to the ground surface, wind speed is affected by the friction force that depends on the terrain. The mean wind velocity profile within the atmospheric boundary layer is described by

$$
U(z)=\frac{1}{k} u_{*} \ln \left(\frac{z}{z_{0}}\right)
$$

in which $u_{*}$ is the friction velocity, $k$ is von Karman's constant $(=0.4), z$ is the height above the ground and $z_{0}$ is the roughness length. The roughness affects the thickness of the boundary layer, and the velocity at any height decreases as surface roughness increases.

In general, the velocity of wind may be represented in vector form as

$$
U(z, t)=U(z) i+u(z, t) i+v(z, t) j+w(z, t) k
$$

where $u, v$, and $w$ are the fluctuating components of gust along the $x$-, $y$-, and $z$-axes (longitudinal, lateral, and vertical axes) and $U(z)$ is the mean wind along the $x$-axis. The fluctuating component along the mean wind direction, $u$, is the largest, and it is therefore the most important parameter pertaining to wind.

The power spectral density (PSD) function is another important function that describes the nature of wind turbulence. To study turbulence using a continuous record of measurements at a single point, we need to assume that the turbulence is frozen at a point. Several mathematical models describe the wind power spectrum. Some of them have been suggested by von Karman, Kolmogoroff, Davenport, Kaimal, etc. For example, the wind speed turbulence spectrum in the along-wind direction was described by Kolmogoroff [18] as:

$$
\frac{n S_{u}(n)}{u_{*}^{2}}=C f^{-2 / 3}
$$

where $n$ is the frequency in $\mathrm{Hz}, C$ is a constant, $u_{*}$ is the friction velocity, $f=n z / U(z)$ is the normalized frequency, and $U(z)$ is the mean wind speed at height $z$.
A general form of the wind turbulence spectrum model was suggested by Olesen et al [18].

$$
\frac{n S_{u}(n)}{u_{*}^{2}}=\frac{A f^{\gamma}}{\left(1+B f^{\alpha}\right)^{\beta}}
$$

The spatial description of turbulence is characterized by a coherence function [19], whereas the turbulence model for a single point in space can be described by Eq. (3) or (4). Turbulence is often associated with wind shear. Wind shear refers to the change in the wind speed or direction with atmospheric elevation.

To describe the characteristics of natural wind, the time history data for the along-wind direction is discussed in this section. The wind speed data were recorded using a hot-wire anemometer at a rate of $1 \mathrm{~Hz}$ with a sampling interval of $1 \mathrm{~s}$. The data contained 1024 data points. We measured the data at a height of $2 \mathrm{~m}$ above the ground in a suburban area. The mean wind speed from the collected data was found to be $3.81 \mathrm{~m} / \mathrm{s}$. That is, $U(2 \mathrm{~m})=3.81 \mathrm{~m} / \mathrm{s}$ and $z_{0}=0.3$. Fig. 1 (a) shows the time history of the measured wind speed and Fig. 1(b) illustrates its power spectrum in a log by log representation.

At first glance, the wind time-series resembles an irregular, noisy mess. However, as shown in Fig. 1(b), the slope of the power spectrum for the actual wind data resembles $1 / \mathrm{f}^{\beta}$-noise when $\beta=1.58(\approx 5.0 / 3.0)$.
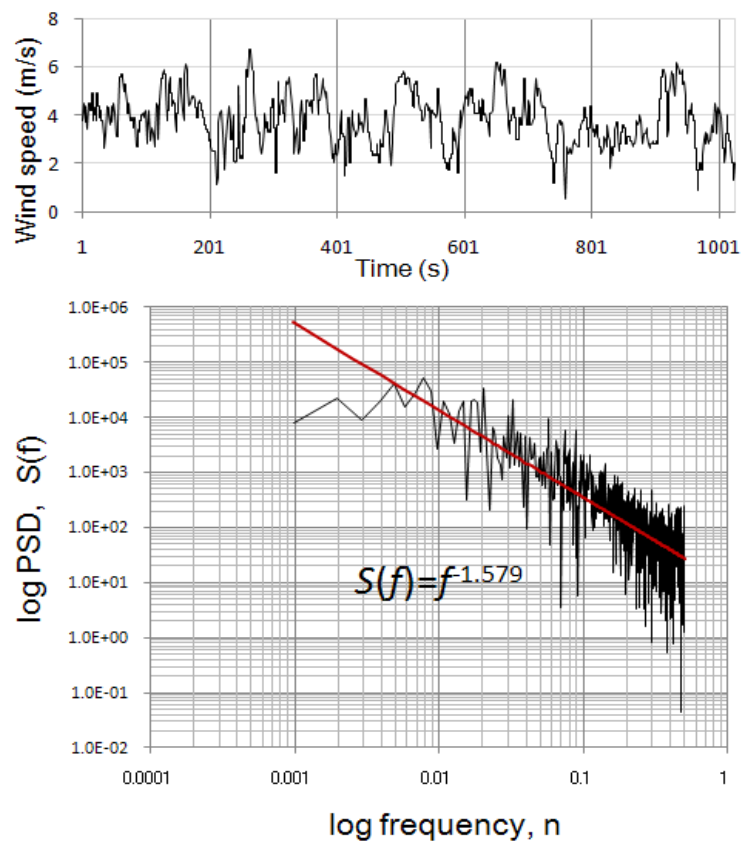

Fig. 1. Measured wind speed history data in the along-mean-wind direction at a point in space and the corresponding power spectrum

Because "1/f-like" noises are quite prevalent in nature, we apply fBm noise while modeling our wind field [14]. 1/f $\mathrm{f}^{\beta}$ noise is an intermediate between white noise with no correlation in time and random walk (Brownian motion) noise with no correlation between increments. In general, a process with a spectral density proportional to $1 / \mathrm{f}^{\beta}$ corresponds to $\mathrm{fBm}$ for which $\beta=2 H+1$. 


$$
S(f)=\frac{1}{f^{\beta}}
$$

where the parameter $\mathrm{H}$ describes small-scale noise roughness.

In the next section, we describe our proposed approach in more detail for constructing a wind field based on fBm noise by considering the characteristics of actual wind.

\section{WIND FIELD MODEL}

\subsection{Comparison with well-known approaches}

Turbulent wind fields are modeled as stochastic processes. Shinya and Fournier [1] employed a stochastic process and applied Fourier synthesis to derive a wind field in the spatiotemporal frequency domain and inverted the result to obtain a periodic space-time wind field. Stam and Fiume [2] employed the same paradigm but they were mainly concerned with coupling the wind field model with microscopic interaction of gaseous and fluid phenomena. In contrast, [1] were mainly concerned with coupling the wind field model with macroscopic physical models of rigid or deformable objects. Both wind field models, however, can be applied to a wide range of phenomena.

Both the methods mentioned above adopted the wind field model used in the structural engineering field; this model considers the characteristics of wind. In [1], Shinya and Fournier used the experimental formulation of the power spectrum of wind in uniform fields and applied Davenport's exponential coherence functions [19] to build a spatially correlated turbulent field. The coherence function is used for all three wind directions. In [1], Shinya and Fournier selected power spectra for each of the following wind components: $u, v$, and $w$. The spectra were respectively referred to as $S_{u}, S_{v}$, and $S_{w}$, and are given by the following equations:

$$
\begin{aligned}
& S_{u}=C_{0} 200(v / f) /(1+50 v)^{5 / 3} \\
& S_{v}=C_{0} 15(v / f) /(1+9.5 v)^{5 / 3} \\
& S_{w}=C_{0} 3.36(v / f) /\left(1+10 v^{5 / 3}\right)
\end{aligned}
$$

where $f$ is the temporal frequency in $\mathrm{Hz}, v=f z / U(z), z$ is the height of the observation point from the ground, and $U(z)$ is the mean wind velocity at that point. To simplify the wind field model, Shinya and Fournier applied Taylor's frozen turbulence hypothesis.

Unlike [1], Stam divided the wind field into a large-scale component and a small-scale component. The small-scale term is modeled by random vector fields by considering the cross-correlation between different components of the velocity field at two different points in space-time. Additionally, the structure of a velocity field is entirely determined by Kolmogoroff's energy spectrum. Moreover, instead of invoking Taylor's hypothesis, Stam and Fuime modeled the temporal frequency according to the energy spectrum function as well as the temporal spread function.

Additionally, the two methods mentioned above [1, 2] produce spatiotemporal wind velocity fields by accounting for spatiotemporal auto-correlation.

\subsection{Important steps in constructing the wind field}

To describe and compare our approach, we outlined the important steps and the differences between the abovementioned approaches [1] and [2].

1. Stam and Fiume [2] assume that a velocity field is homogenous in space and stationary in time, which implies that the corresponding cross correlation only depends on the difference between the two points and the difference between the two times.

2. Stam and Fiume [2] assume that the velocity field is spatially isotropic. Thus, the cross-correlation functions only depend on the distance between the points.

3. The structure of a velocity field in [2] is determined by its energy spectrum function (via its cross-spectral density functions).

4. The approach of Stam and Fiume [2] uses the best-known Kolmogoroff energy spectrum, whereas Shinya and Fournier use the wind turbulence spectrum model, which is determined using experimental data and given by Eq. (6).

5. Instead of invoking Taylor's hypothesis [1], Stam and Fiume model the temporal frequency dependence of the energy spectrum using a temporal spread function.

6. Before computing the velocity field in the frequency domain, Shinya and Fournier [1] implement Fourier transform to reduce spatial dependence, whereas Stam and Fiume [2] compute transformed kernels in terms of cross-spectral density functions by inserting expressions for Fourier velocity components.

7. The velocity field is then obtained by taking the inverse Fourier transform for each wind component.

\section{OUR APPROACH}

Our fBm-based wind field model does not cover cross-spectral density functions applied in the approaches [1, 2] mentioned in the previous section. We construct the wind field directly from the power spectral density functions by applying higher dimensional $\mathrm{fBm}$ noise. Therefore, our approach is quite simple as compared to the existing approaches.

Additionally, we consider the key characteristics of natural wind employed in the structural engineering field in our wind field modeling approach. Our approach provides an intuitive description of wind parameters and allows animators to control the wind field by using those parameters. It also constructs spatially as well as temporally varying 2D and 3D wind fields and shows a comparison of the visualization results of the two approaches mentioned above.

\subsection{Consideration of power spectral density}

Since we use higher dimensional fBm noise data, we employ the PSD function, which is given as follows. 


$$
S\left(f_{1}, \ldots, f_{n}\right)=\frac{A}{\left(\sum_{i=1}^{n} f_{i}^{2}\right)^{\beta+(n-1)}}
$$

where $n$ is the dimension of the wind field and $\beta$ is the slope of the power spectrum. The algorithm developed by Voss [20] is the main point of reference for the generation of $1 / \mathrm{f}$ noise.

\subsection{Physical meanings of wind model field model parameters}

If we adopt the wind power spectrum model suggested by Kolmogoroff and given by Eq. (3), the intuitive meanings of parameters $A$ and $\beta$ can be derived from the following equation:

$$
S_{u}(n)=u_{*}^{2}\left(\frac{U(z)}{z}\right)^{\frac{2.0}{3.0}} \frac{C}{n^{5 / 3}}
$$

In our model, we determined the parameter values of $A$ and $\beta$ with small modifications, given as follows:

$$
A_{u}=u_{*}\left(\frac{U(z)}{z}\right)^{\frac{2.0}{3.0}} \quad \beta=\frac{5.0}{3.0}
$$

When we used the wind turbulence spectrum model, we assumed that the constant $C$ is equal to 1 and omitted the degree of the power of friction velocity because our model was highly sensitive when we presumed the friction velocity as a degree of power, as defined in other wind field models. Usually wind spectrum models describe strong wind turbulence at high altitudes. However, in outdoor scenery simulations, we need to simulate winds closer to the ground.

From Eq. (9), we can describe the physical meaning of a parameter $\mathrm{A}$ in the along-mean-wind direction in terms of wind characteristics, where $z$ is the observed height above the ground, $u_{*}$ is the friction velocity, and $U(z)$ is the mean wind velocity at a height of $\mathrm{z}$.

The lateral and vertical turbulence components are generally lower in magnitude than the corresponding longitudinal value. Thus, the following simple relationships are applied to the parameter value of $\mathrm{A}$ for the other two directions of wind velocity [21]:

$$
\begin{aligned}
& A_{v}=0.88 A_{u} \\
& A_{w}=0.55 A_{u}
\end{aligned}
$$

The parameter $\beta$ can vary depending on the characteristics of wind as well as the velocity component of wind. Generally, the parameter $\beta$ is selected as 5.0/3.0 according to Kolmogoroff's law applied in the along-mean-wind direction. Also, the friction force $u_{*}$ can be derived from Eq. (1). To determine the friction force $u *$ we must know the mean wind speed $U(z)$ at a height of $z$ above the ground and the terrain roughness $z_{0}$. Typical values of terrain are provided in TABLE 1 . Animators can use TABLE 1 as the reference to control the wind field while simulating a specific type of wind depending on the terrain roughness and the height of the observation point. In section IV, we illustrate the influence of parameters on the simulated wind fields for various terrain conditions at different heights.

\subsection{Wind shear}

As mentioned in section I, the mean wind speed is characterized by terrain roughness, height of the observation point, and friction velocity. Surface friction slows down wind speed and changes the wind direction. Because of wind shear, the wind speed or direction changes with height in the atmosphere.

TABLE 1: VALUES OF ROUGHNESS LENGTH $z_{0}$

\begin{tabular}{|l|c|}
\hline \multicolumn{1}{|c|}{ Type of terrain } & $z_{0}(\mathrm{~m})$ \\
\hline Sand & $0.0001-0.001$ \\
\hline Sea surface & 0.005 \\
\hline Grass & $0.01-0.1$ \\
\hline Pine forest & $0.90-1.0$ \\
\hline Suburban areas & $0.20-0.40$ \\
\hline Centers of cities & $0.35-0.80$ \\
\hline
\end{tabular}

\subsection{Taylor's frozen turbulence hypothesis}

We can use Taylor's frozen turbulence hypothesis to interpret time series as a space series, as used by [1]. Taylor's hypothesis is expressed as

$$
u(x, y, z, t)=u(x-t U, y, z)
$$

The advantage of using this hypothesis is that it enables dimensional reduction. Thus, we applied this hypothesis to the two-dimensional frozen wind fields in our grass field simulation.

\subsection{Implementation steps}

We now describe the implementation steps involved in the calculations made in our proposed approach.

Step 1: Determine characteristics of wind fields by defining wind parameters such as mean wind speed $U(z)$ at the given height $z$ and the terrain roughness $z_{0}$.

Step 2: Calculate friction force using Eq. (1).

Step 3: Calculate parameter values of $A_{u}, A_{v}$, and $A_{w}$, as defined in section IV. 2, for each wind velocity component.

Step 4: Select parameter $\beta$ as 5.0/3.0 according to Kolmogoroff's law applied in the along-mean-wind direction.

Step 5: Construct wind power spectrums for each wind direction according to Eqs. (7), (9) and (10).

$$
\begin{aligned}
& S_{u}\left(f_{1}, \ldots, f_{n}\right)=\frac{A_{u}}{\left(\sum_{i=1}^{n} f_{i}^{2}\right)^{\beta_{u}+(n-1)}} \\
& S_{v}\left(f_{1}, \ldots, f_{n}\right)=\frac{A_{v}}{\left(\sum_{i=1}^{n} f_{i}^{2}\right)^{\beta_{v}+(n-1)}}
\end{aligned}
$$




$$
S_{w}\left(f_{1}, \ldots, f_{n}\right)=\frac{A_{w}}{\left(\sum_{i=1}^{n} f_{i}^{2}\right)^{\beta_{w}+(n-1)}}
$$

Step 6: Apply inverse Fourier transform based on the dimension of the simulated wind field for each wind component:

\section{$\operatorname{IFFTnD}\left(S_{u}\right)$ \\ $\operatorname{IFFTnD}\left(S_{v}\right)$ \\ $\operatorname{IFFTnD}\left(S_{w}\right)$}

Step 7: Employ Taylor's frozen turbulence hypothesis to generate frozen wind fields, if required.

\section{RESULTS}

\subsection{Experimental results}

This section briefly describes the experimental results obtained by constructing specific types of wind fields according to the terrain and height pertaining to the selected parameter values that are listed in TABLE 1 , as illustrated in section IV. 4.

In our experiments, we simulated winds for different terrains. In the first case, we constructed winds in suburban area. In the second case we considered terrain as an open area. In our simulations we also examined the elevation of the observation points. This could be used in graphical outdoor scenery simulations. The simulated winds are illustrated in Fig. 2 with the selected wind parameters. As shown in Fig. 2, wind turbulence has a strong dependence on terrain roughness and the elevation of the observation point for a given mean wind speed. Wind is more turbulent in suburban areas than in open areas.

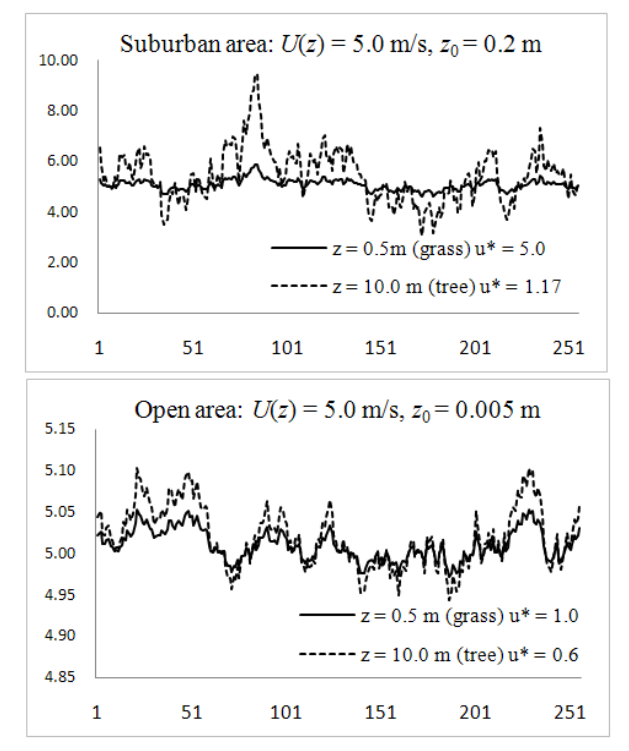

Fig. 2. Simulated winds at different parameter values

\subsection{Wind field simulation and its visualization}

In this section, we illustrate the simulation of 3D non-frozen turbulence and 2D frozen turbulence wind fields and compare our results with those obtained by other well-known approaches. To demonstrate the turbulence of 3D velocity fields, we used an RGB color combination that corresponds to the magnitude of the velocity components $u, v$, and $w$. For example, the color value $\mathrm{R}$ determines the strength of the $\mathrm{u}$ component in the simulated wind field at a given point. Further, in our illustration, the direction as well as magnitude of velocity is depicted by a line (see Fig. 3).
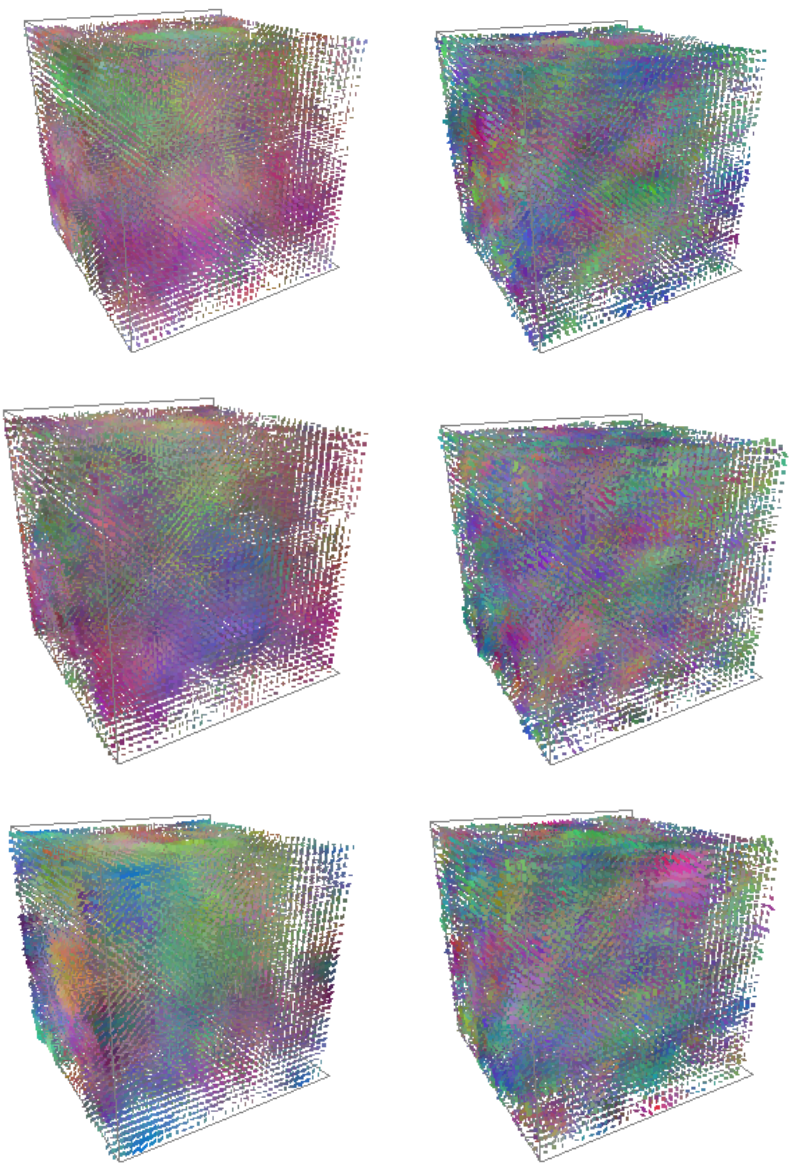

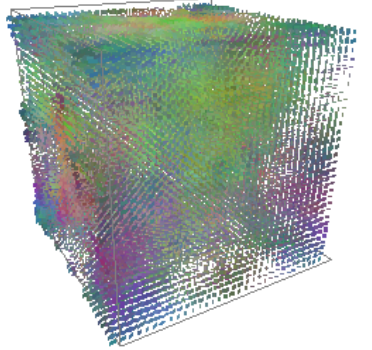

(a)

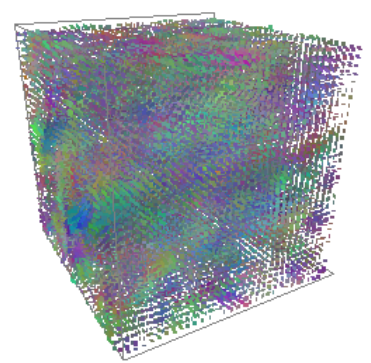

(b)
Fig. 3. Three-dimensional non-frozen turbulent wind fields $(32 \times 32 \times 32 \times 32)$. Screenshots of simulated three-dimensional wind velocity fields. (a) By our approach. (b) By Stam and Fiume’s approach [2]. 

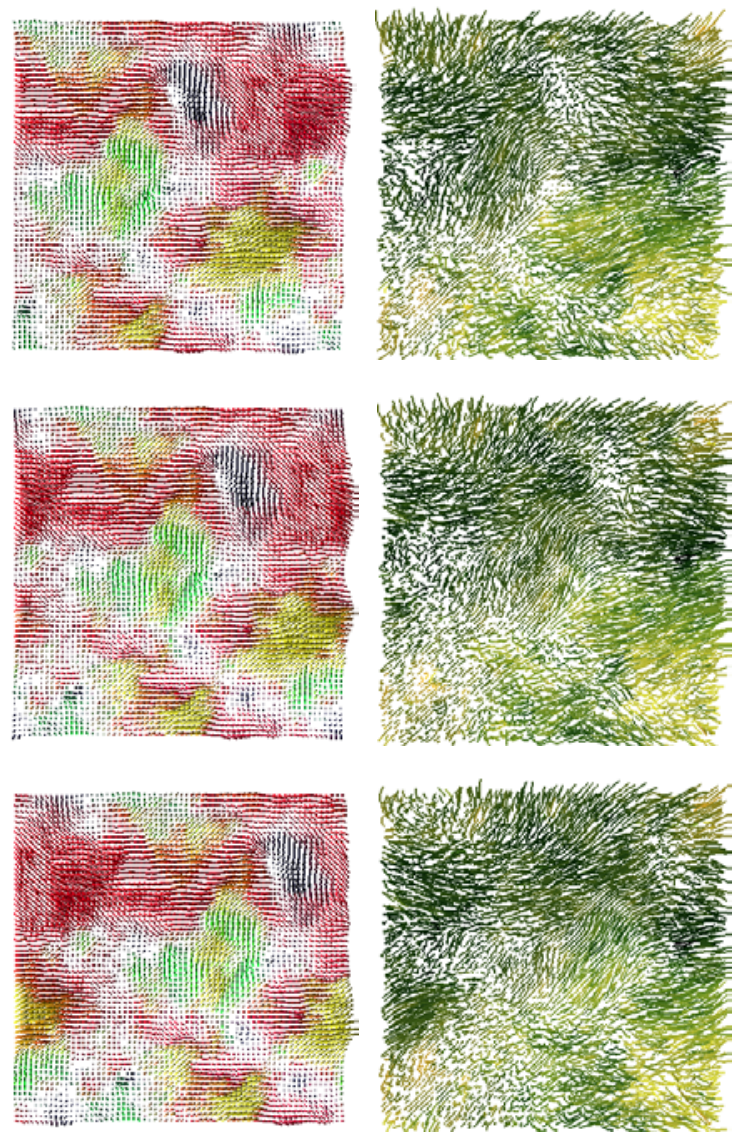

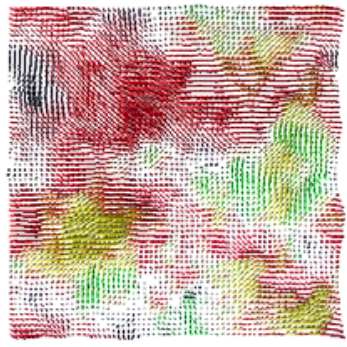

(a)

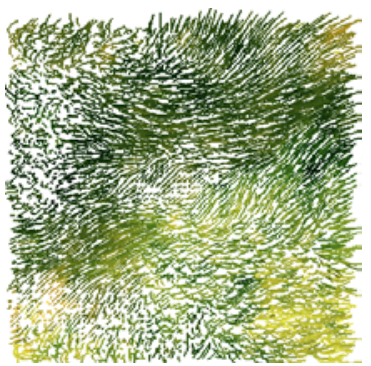

(b)
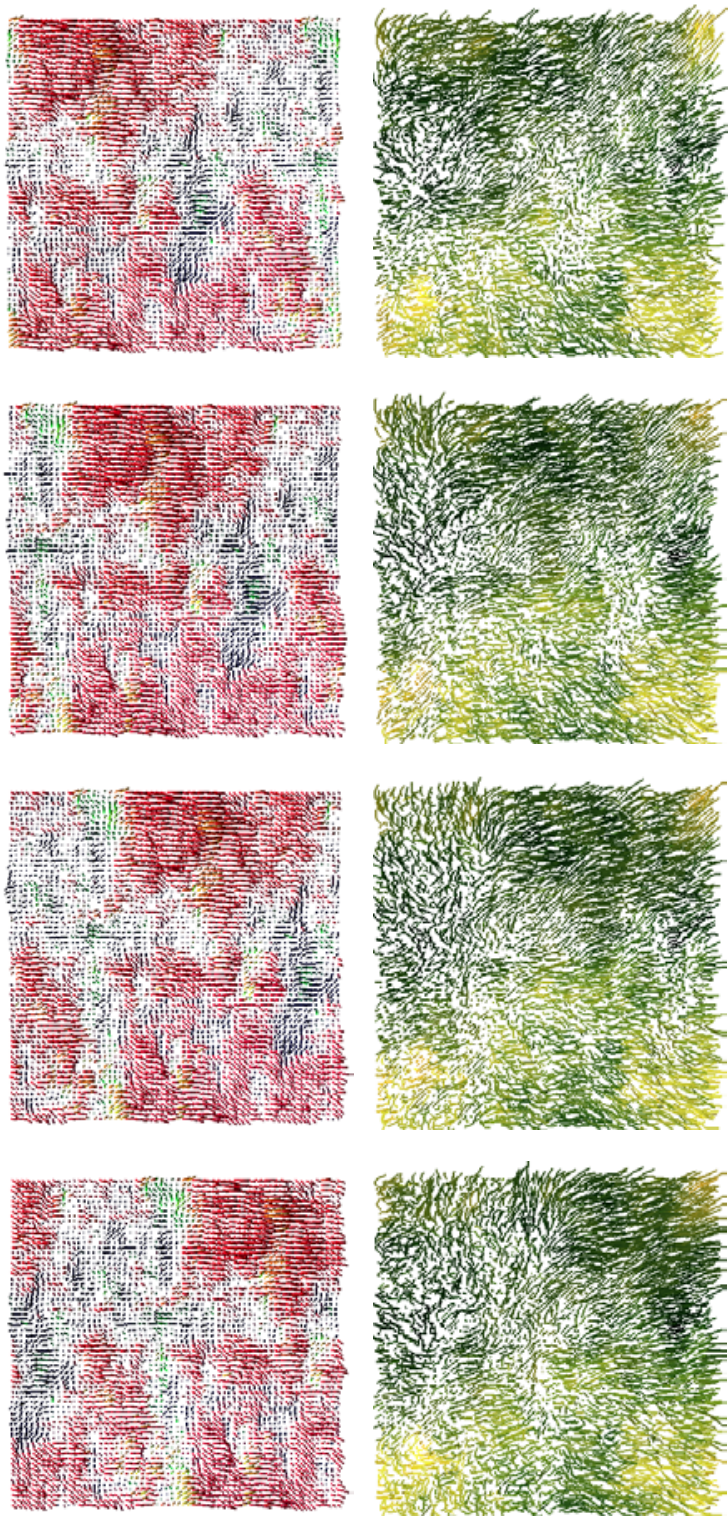

(a)
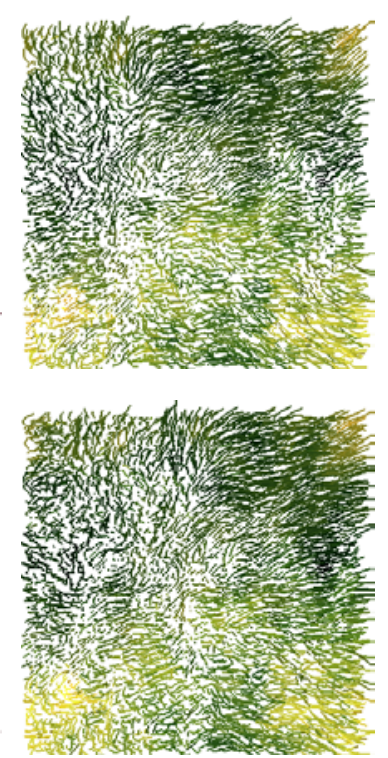

(b)
Fig. 4. 2D frozen turbulent wind fields $(64 \times 64)$.

(a) Screenshots of simulated 2D wind velocity fields by our approach. (b) Grass fields generated by the corresponding 2D wind fields.

We chose a grass field to visualize two-dimensional wind fields and it is flexible and therefore most suitable for simulation. In our grass field simulation, we considered a simple object that modeled individual blades of grass and employed the wind velocity field simulated by our proposed approach. For large-scale scenery simulations such as those of grass fields, it is better to use Taylor's frozen turbulence hypothesis to decrease the dimensionality of space.

We compared the result of our grass field simulation and its wind velocity field with the results obtained from Shinya and Fournier's approach (see Fig. 4 and Fig. 5). From the experiments, our simulated grass field illustrates effects that are very similar to natural grass fields as well as the simulated fields obtained by Shinya's approach.
Fig. 5. 2D frozen turbulent wind fields $(64 \times 64)$.

(a) Screenshots of simulated 2D wind velocity fields by Shinya and Fournier's approach [1]. (b) Grass fields generated by the corresponding 2D wind fields.

We preferred tracing particles through the wind fields as a better visualization method for 3D wind fields. Fig. 3 shows screenshots of 3D wind fields implemented by our approach; they are compared with those implemented by Stam's approach. We compared our results for particle tracing to the ones obtained by Stam and Fiume's approach. The implementation results are shown in Fig. 6 and Fig. 7. TABLEs 2 and 3 show a comparison between our approach and other approaches in terms of method complexity and computational cost.

TABLE 2: PERMORMANCE RESULTS OF 2D FROZEN TURBULENT WIND FIELDS.

\begin{tabular}{|l|c|c|}
\hline & Size $512 \times 512$ & Size $1024 \times 1024$ \\
\hline Our approach & $359 \mathrm{~ms}$ & $2,438 \mathrm{~ms}$ \\
\hline Shinya et al. [1] & $493 \mathrm{~ms}$ & $2,969 \mathrm{~ms}$ \\
\hline
\end{tabular}



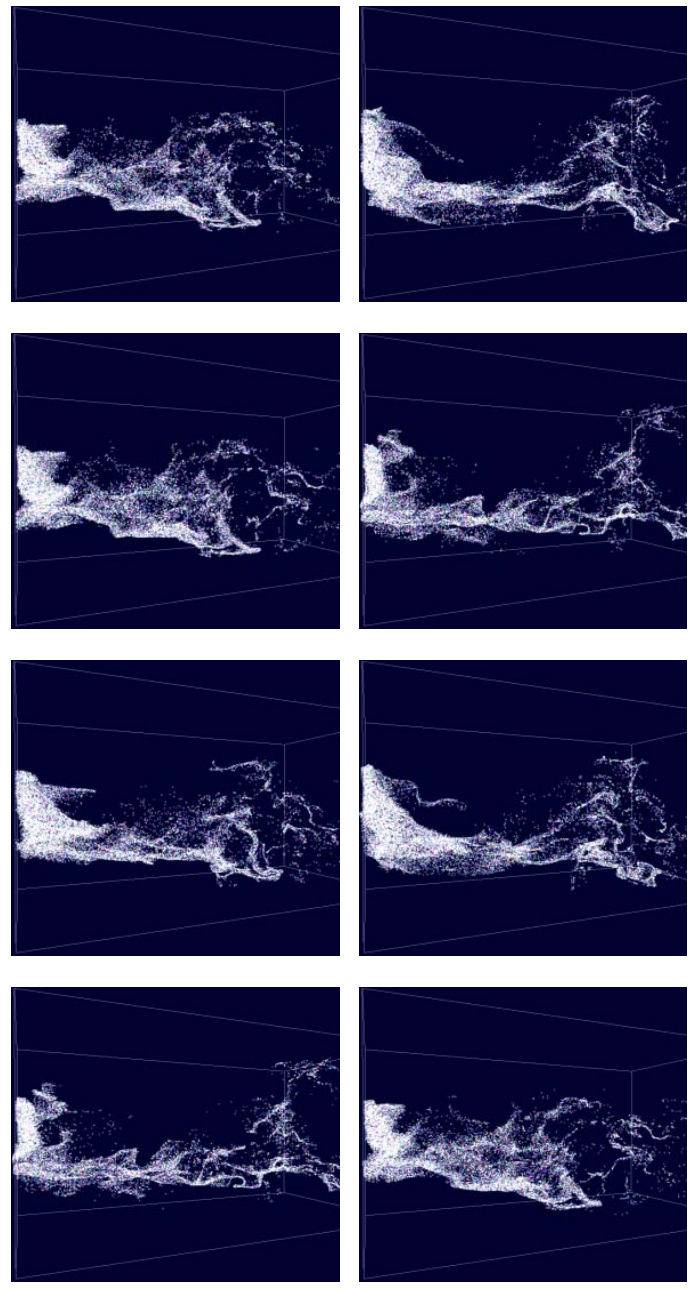

Fig. 6. Three-dimensional non-frozen turbulent wind fields $(64 \times 32 \times 32 \times 64)$ Visualization by our approach.

TABLE 3: PERMORMANCE RESULTS OF 3D NON-FROZEN TURBULENT WIND FIELDS.

\begin{tabular}{|l|c|c|}
\hline & $32 \times 32 \times 32 \times 32$ & $64 \times 64 \times 64 \times 64$ \\
\hline Our approach & $3,250 \mathrm{~ms}$ & $64,297 \mathrm{~ms}$ \\
\hline Stam et al. [2] & $4,188 \mathrm{~ms}$ & $83,158 \mathrm{~ms}$ \\
\hline
\end{tabular}

Note that performance measurements were obtained using a computer with Intel Core $(3.0 \mathrm{GHz})$ processor and 2GB of RAM and measured in milliseconds.

\section{CONCLUSION}

We presented an alternative approach to simulate three-dimensional non-frozen turbulent wind fields. By using an $\mathrm{fBm}$-noise based approach with the characteristics of natural wind, wind fields can be constructed in a simple manner with reasonable visual accuracy. Simulated wind fields are described by relatively few parameters. Our approach provides an intuitive description of wind parameters to control wind fields. The implementation results demonstrate that our proposed approach is competitive with other well-known approaches through the comparison of implementation results and visualizations.
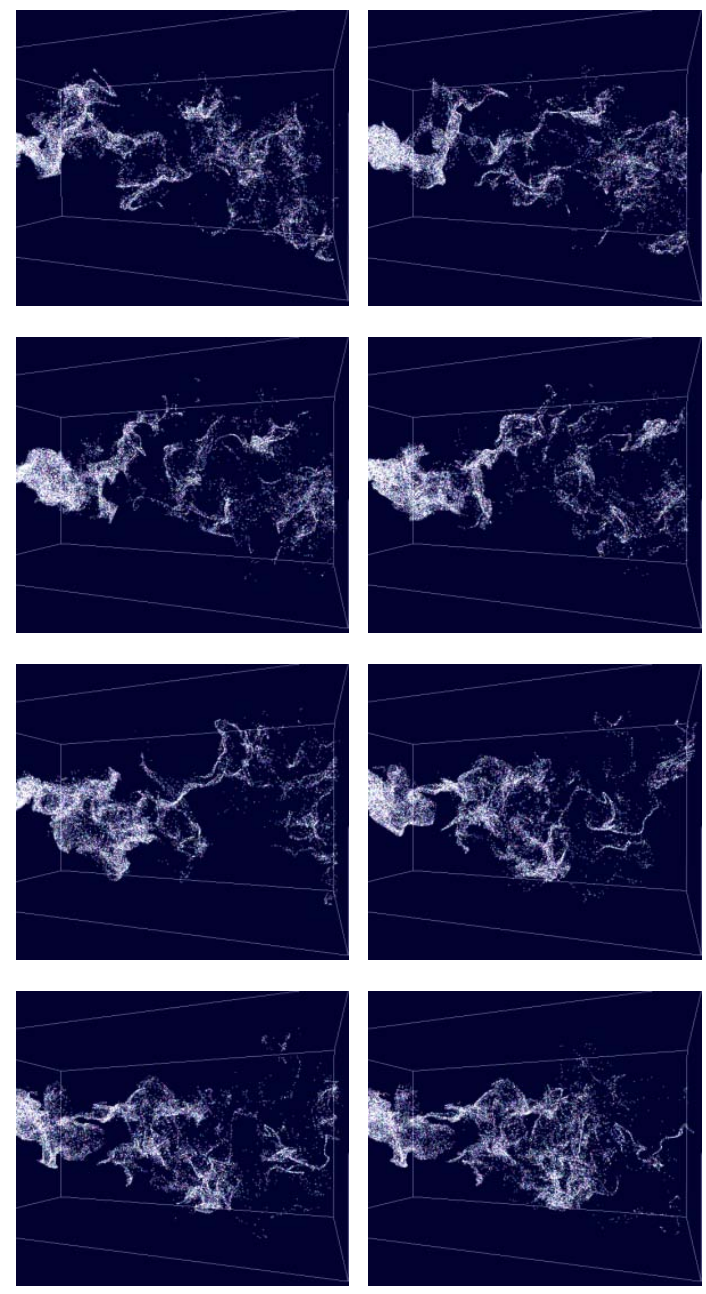

Fig. 7. Three-dimensional non-frozen turbulent wind fields $(64 \times 32 \times 32 \times 64)$. Visualization by Stam and Fiume’s approach [2]

\section{ACKNOWLEDGEMENT}

This work was partially supported by the research grant from TOSTEM Foundation for Construction Materials Industry Promotion.

\section{REFERENCES}

[1] M. Shinya and A. Fournier. Stochastic motion - motion under the influence of wind, in Proceedings of Eurographics '92, pp. 119-128, 1992.

[2] J. Stam and E. Fiume. Turbulent wind fields for gaseous phenomena, in SIGGRAPH 93 Conference Proceedings, Annual Conference Series, pp. 369-376, 1993.

[3] S. Ota, M. Tamura, T. Fujimoto, K. Muraoka and N. Chiba. A hybrid method for real-time animation of trees swaying in wind fields, The Visual Computer, vol. 20, pp. 613-623, 2004.

[4] S. Hu, T. Fujimoto, N. Chiba. Pseudo-dynamics model of a cantilever beam for animating flexible leaves and branches in wind field, Computer Animation and Virtual Worlds, pp. 279-287, 2009.

[5] J. Stam. Stable fluids, in SIGGRAPH 99 Conference Proceedings, Annual Conference Series, pp. 121-128, 1999. 
[6] N. Foster and D. Metaxas. Modeling the motion of a hot, turbulent gas, in Computer Graphics Proceedings, Annual Conference Series, pp. 181-188, 1997.

[7] J. Wejchert and D. Haumann. Animation Aerodynamics, ACM Computer Graphics (SIGGRAPH '91), 25(4), pp.19-22, 1991.

[8] J. Stam. Stochastic dynamics: Simulating the effects of turbulence on flexible structures, Computer Graphics Forum, 16(3), pp. 159-164, 1997.

[9] M. Sun, A. D. Jepson, E. Fiume. Video input driven animation (VIDA), in Proceedings of IEEE International Conference on Computer Vision (ICVV), pp. 96-103, 2003.

[10] Y.-Y. Chuang, D. B. Goldman, K. C. Zheng, B. Curless, D. H. Salesin and R. Szeliski. Animation pictures with Stochastic Motion Textures, $A C M$ Trans. Graph., 24(3), pp. 853-860, 2005.

[11] R. Habel, A. Kustering, M. Wimmer. Physically Guided Animation of Trees, Computer Graphics Forum, vol. 28, pp.523-532, 2009.

[12] T.Fujimoto, S.Miyauchi, T.Suzuki and N.Chiba. Noise-based Animation of Waving Phenomena, Proc. IWAIT2005, pp.459-464, (IEICE technical report. Image engineering, 104(545), pp.93-98), 2005.1

[13] W. Qiang, Y. Zheng, C. Chun, T. Suzuki, T. Fujimoto and N. Chiba. Efficient Rendering of Breaking Waves Using MPS Method, Journal of Zhejiang University SCIENCE A, 7(6), pp.1018-1025, 2006

[14] B. B. Mandelbrot, The Fractal Geometry of Nature, W. H. Freeman and Company, New York, 1977.

[15] H.O. Peitgen and D. Saupe. The Science of Fractal Images, Heinz-Otto and Dietmar Saupe. Springer-Verlag, New York, Inc., 1988.

[16] K. Perlin. An Image Synthesizer, in Proceedings of SIGGRAPH 1985, pp. 287-296, 1985.

[17] A. Lagae, S. Lefebvre, R. Cook, T. DeRose, G. Drettakis, D. S. Ebert, J. P. Lewis, K. Perlin and M. Zwicker. A survey on procedural noise functions, Computer Graphics Forum, 29(8), pp. 2579-2600, 2010.

[18] H. R. Olesen, S. E. Larsen and J. Højstrup. Modelling velocity spectra in the lower part of the planetary boundary layer, Boundary-Layer Meteorology, 29(3), pp. 285-312, 1984.

[19] K. Saranyasoontorn, L. Manuel and P. S. Veers. A comparison of standard coherence models for inflow turbulence with estimates from field measurements, Journal of Solar Energy Engineering, 126(4), pp. 1069-1082, 2004.

[20] R. F. Voss. Fractals in nature: From characterization to simulation. in The Science of Fractal Images, Springer-Verlag, New York, Inc. pp. 21-70, 1988

[21] J. D. Holmes. Wind Loading of Structures, Spon Press, London, 2001.

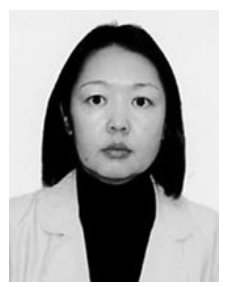

Oyundolgor Khorloo received a BS degree in applied mathematics from National University of Mongolia in 1995 and an MS degree in computer science from University of Colorado at Denver in 2001. She is currently a $\mathrm{PhD}$ candidate in computer science at Iwate University. Her research interests include computer graphics, noise-based animation and simulation of natural phenomena.

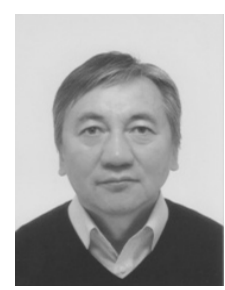

Zorig Gunjee is a researcher in ECM Co., Ltd, Mongolia. He received his bachelor's and master's degrees from Novosibirsk Technical University, Russia in 1986. He also got his master's degree in computer science from Mongolian University of Science and Technology in 2001 and his PhD degree from Iwate University, Japan in 2007. His research areas include point rendering, noise based animation, computer animation of natural phenomena, and graphics processing unit (GPU)

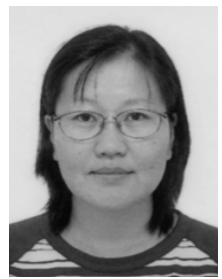

Batjargal Sosorbaram is currently a research fellow in the Department of Computer Science at Iwate University. Her research interests include computer graphics and noise-based animation. She received a BE in science of physics from National University of Mongolia in 1988 and an ME and DE degrees in computer science from Iwate University in 1999 and 2003, respectively.

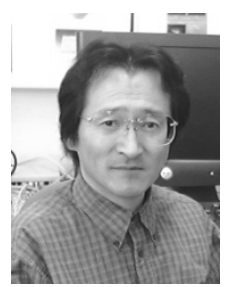

Norishige Chiba is currently a Professor in Department of Computer Science at Iwate University. His research interests include Computer Graphics, Laser Graphics and Interactive Graphics. He received a $\mathrm{BE}$ in electrical engineering from Iwate University and an ME and DE in information engineering from Tohoku University in 1975, 1981 and 1984, respectively. He worked at Nippon Business Consultant Co., Ltd from 1975 to 1978. He was a research associate in the Department of Communication Engineering at Tohoku University from 1984 to 1986, an associate professor of Computer Science at Sendai National College of Technology from 1986 to 1987 and an associate professor of Computer Science at Iwate University from 1987 to 1991. He is a member of The Society for Art and Science, IEICE, IPS, IEEE and ACM. 